\title{
Method for effective monitoring and control of the positioning of the output link of the mechatronic module
}

\author{
Eugene Lavrentjev, Anastasia Sergienko, Andrei Shevyrev and Ekaterina Vasilyeva \\ Don state technical University, Rostov-on-don, Russia
}

\begin{abstract}
This article is devoted to the problem of linear positioning of the output link of a mechatronic module (MM), made on the basis of a ball screw (ball screw). The analysis of typical schemes for constructing an $\mathrm{MM}$ controller using signals from radial position sensors of the ball screw rotor and a discrete indicator of the linear position of the output link in the working stroke section with the possibility of implementing an emergency (emergency) power off of the entire positioning system in the event of the output link extending beyond the working stroke. An optimal technical solution is proposed for constructing a linear positioning sensor of the output link based on combining the functions of the "referent points" indicator and the "limit switch" MM. The cost-effectiveness of the new
\end{abstract}

\section{Introduction}

In the modern technological world, mechatronical modules [1] are increasingly used for performing fast and accurate various actuators automated movements in such economic sectors as: precision engineering and automotive; machine tools and equipment for the automation of technological processes; aviation, space and military equipment; office and computer equipment; office and computer equipment; micromechanics and robotics; medical equipment; agriculture [2], etc. The criterion for the quality of work (movement) of actuators in mechatronic modules is the problem orientation of the output link, which is determined by a specific applied task.

The criterion of performance (motion) in the mechatronic actuators modules is problematic orientation of the output link defined by the particular application.

For MMs made on the basis of ball screws, along with the achievement of the constructive compactness of the modules and the improvement of the weight and size, dynamic characteristics of the machines due to the simplification of the kinematic chains, such tasks are:

For MM executed based leadscrew, while achieving structural compactness modules and improving mass and size, the dynamic characteristics of machines due to simplification of the kinematic chains, such tasks are:

- ensuring the specified accuracy of the linear positioning of the output link within the working stroke with the possibility of emergency (emergency) power off the entire installation in the event of an abnormal movement of the output link beyond the working stroke; 
- ensuring high reliability (resistance to failures) and noise immunity of electronic equipment by applying a minimum (necessary and sufficient) set of used sensors and switches of electrical circuits.

- providing a predetermined positioning accuracy of the linear output unit within the power stroke, with the emergency (emergency) power switching entire plant in case of abnormal movement output element beyond the working stroke;

- ensuring high reliability (resistance to failures) and noise immunity of electronic equipment with a minimum (necessary and sufficient) set of used sensors and switches of electrical circuits.

- providing a high reliability (fault tolerance) and noise immunity of electronic equipment with minimal (necessary and sufficient) a set of sensors used and commutators of electric circuits.

\section{Materials and methods}

Since the basic MM actuator is a ball screw, which converts the rotational motion of the ball screw nut, rigidly fixed to the rotor of the MM electric motor, into the translational movement of the output link (carriage), then, as a rule, the following are used to monitor and control the positioning of the MM output link:

Since the actuator base is MM ball screw, which provides transformation of rotational motion ball screw nut is rigidly fixed to the rotor of the motor MM into linear motion output member (carriage), for monitoring and control of the output link MM positioning is generally used:

- one incremental encoder [5,6] that registers the current radial position of the ball screw nut (angle of rotation and the number of full turns of the nut relative to the reference "zero" point of the counting origin);

- one incremental encoder (encoder) that registers the current radial position of the ball screw nut (angle of rotation and the number of full turns of the nut relative to the reference "zero" point of the counting origin);

- two discrete sensors [7] (microswitches) signaling the actual achievement by the MM output link of the points of the beginning and end of the working stroke on the linear section of the movement;

- one, at least, a sensor (limit switch) [8,9], signaling the emergency extension of the output link beyond the working stroke with the function of emergency automatic shutdown of the power supply of the entire MM power plant.

- one, at least, a sensor (limit switch), signaling the emergency extension of the output link beyond the working stroke with the function of emergency automatic shutdown of the power supply of the entire MM power plant.

- one, at a minimum, the sensor (limit switch), signaling an emergency nomination output link beyond the working stroke with the function of emergency automatic power off all of the power plant MM.

As an encoder, an optocoupler of the visible or infrared range with an open optical channel is usually used, in the form of an air gap between the LED and the phototransistor.

As commonly used photocoupler encoder visible or infrared optic with an open channel in the form of an air gap between the LED and phototransistor.

The principle of its use to determine the radial position of the ball screw nut in $\mathrm{MM}$ is shown in Figure 1 [2]. 


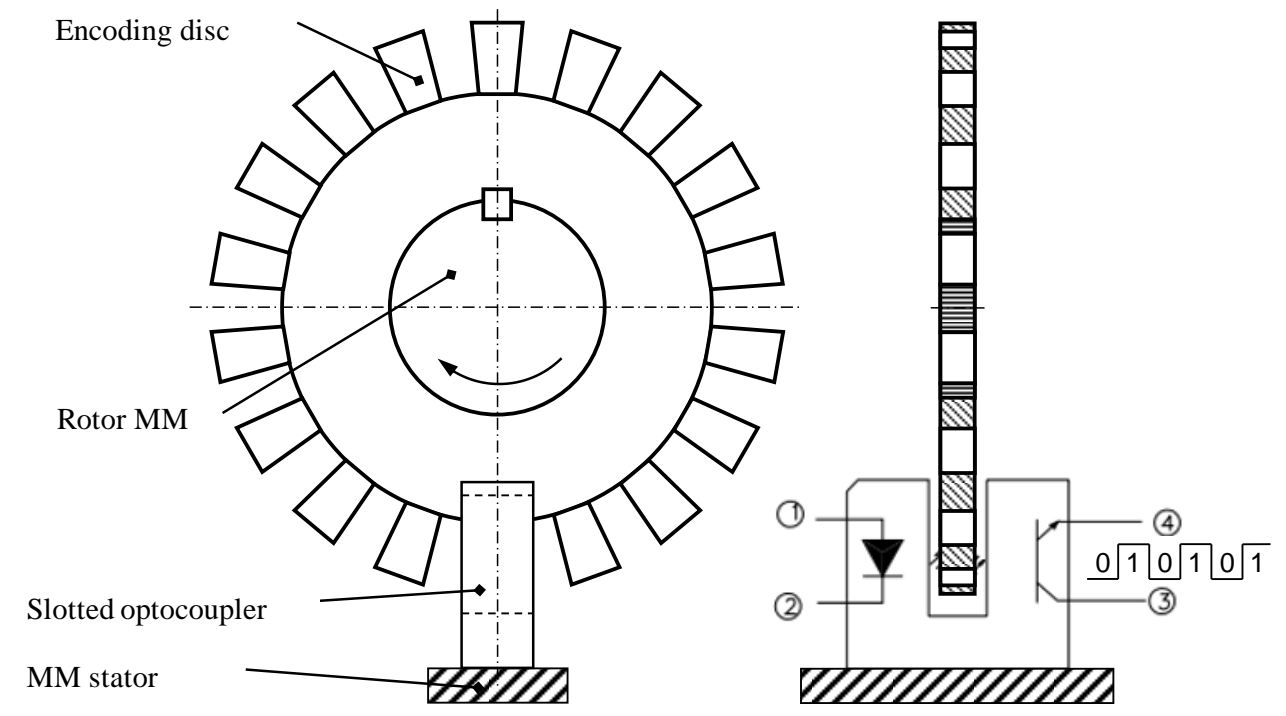

Fig. 1. Principle of measuring the radial position of the ball screw nut in MM

Microswitches with a roller pusher lever, for example, such as shown in Figure 2, are usually used as discrete sensors signaling the actual achievement of the MM output link of the start and end points of the working stroke on the linear section of movement.

Their presence in the controller circuit is justified by the fact that for the practical application of MM, information alone about the relative displacement of the executive link is not enough. It is necessary to know exactly relative to which reference point on the working section of the path these measurements were obtained, which is easiest to perform with a microswitch. In other words, to control the operation of the MM, the controller must "be able" to determine the absolute position of the ball screw nut on the working length of the ball screw in linear expression. For this, the microswitch is placed on the output link or on the MM carriage, and a control stop (protrusion) is installed on the working section of the path in a predetermined place.
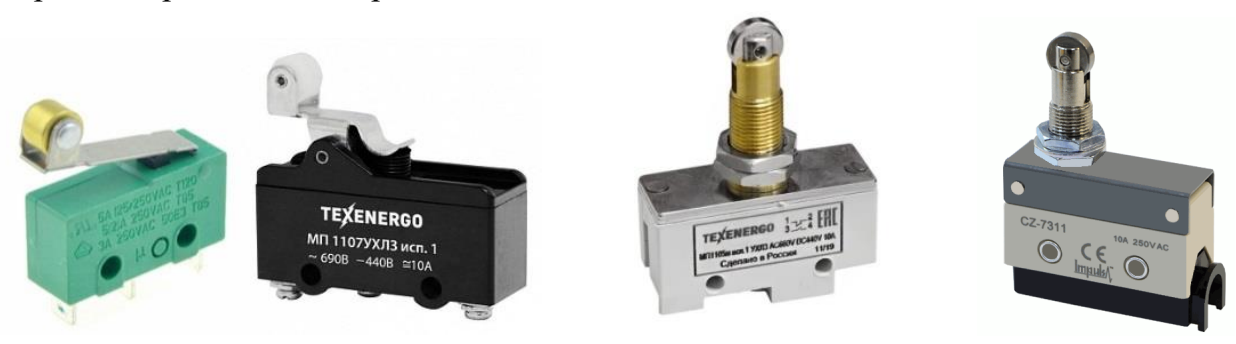

Fig. 2. Samples of microswitches with a roller pusher lever

During the movement of the MM along the ball screw axis when the roller pusher lever hits the control stop, the microswitch is triggered and the signal is sent to the controller, which will perceive it as a pointer to the reference point to set the "zero" count of the summing optical rotation sensor (encoder). The location of the control stop, which is known in advance for the controller, even at one single reference point, allows it to perform and control the reciprocating movements of the MM actuator in the range of the working stroke, preventing it from moving beyond the permissible limits. 
The main disadvantage of this model is the loss of incremental angular information during power failures and, as a consequence, the loss of "orientation" (direction) to a single reference point after power is restored.

The only solution for reliable start and stop of the encoder after power failures, as well as at the initial power up, is to use at least two discrete sensors (microswitches) with actuation points (location of control stops) at the edges of the MM working stroke (one for each sensor), which shown in Figure 3.

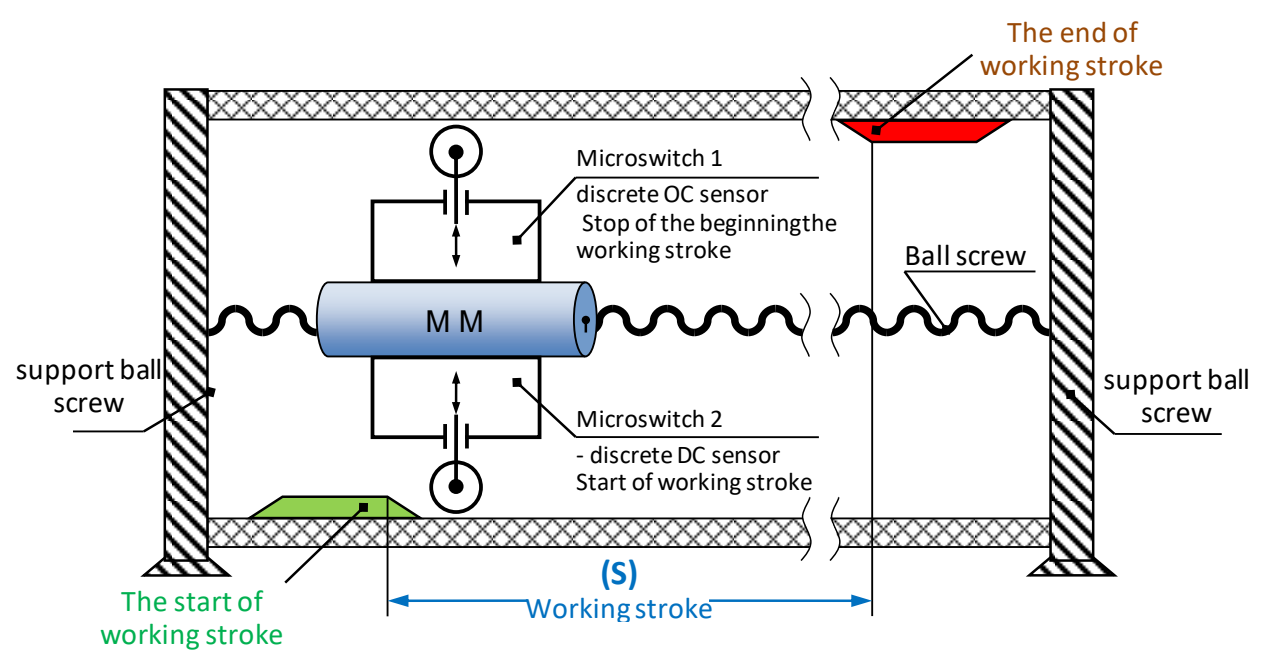

Fig. 3. A variant of the layout of the MM with two microswitches discrete sensors of reference points "Start of the working stroke" and "End of the working stroke"

In order to avoid possible destructive consequences of abnormal extension of the output link beyond the working stroke, a limit switch is a mandatory element of the electrical circuit of any unit performing the reciprocating movements of the output link (Figure 4).

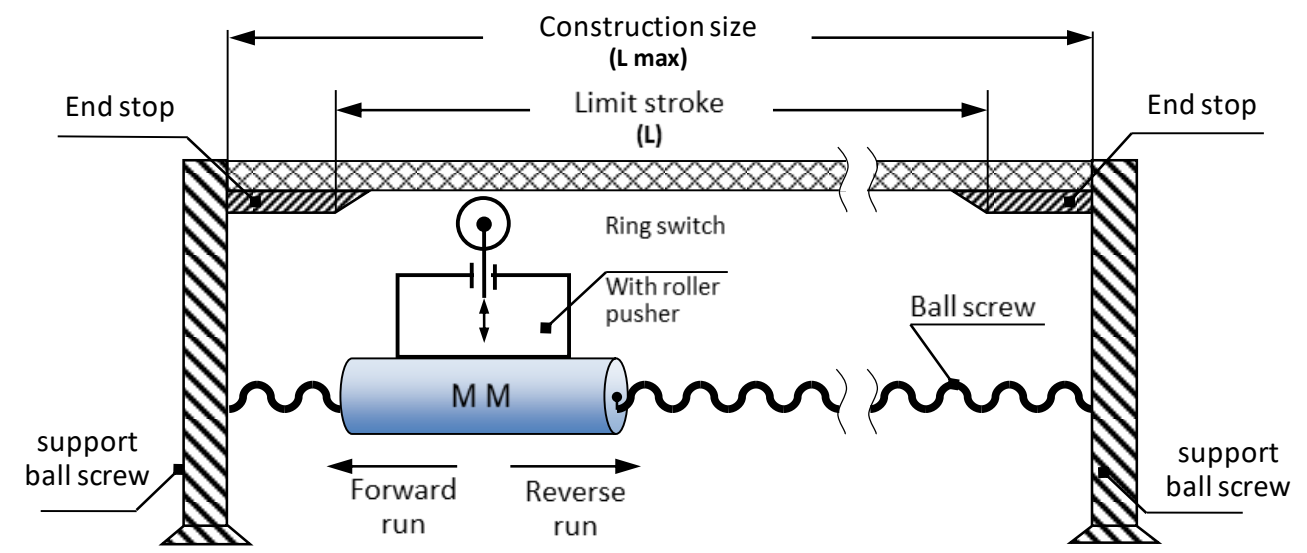

Fig. 4. Variant of arrangement of MM with a roller-type limit switch

It is a power switch with one (or more) normally closed contacts, through which the entire power plant is powered. The main task of the limit switch is to signal when the object has reached a critical point of the way, and, at the same time, as an actuator, to 
automatically open the electrical circuit of the power plant. It is the second function (automatic opening of the electrical circuit of the power plant), as a rule, that limits the possibilities of using the limit switch as a full-fledged sensor for reading its signal by the controller. Since opening the electrical circuit of the entire power plant is an emergency measure, the power to the controller will also automatically turn off even before it has time to process this signal and issue a control command to the electric motor.

Thus, the analysis of standard solutions for constructing an MM based on ball screws showed that the following are used to control and measure the linear displacements of the output link in reciprocating mechanisms:

1) a sensor for the radial position of the rotor MM together with at least two discrete sensors (microswitches) signaling that the MM actuator reaches the reference points "start of the working stroke" and "end of the working stroke" on the linear section of the movement of the MM;

2) at least one limit switch (for example, roller type), which automatically opens the electrical circuit of the entire power plant to prevent possible destructive consequences of an abnormal extension of the MM output link beyond the design restrictions on the linear section of the MM movement.

\section{Results}

The only drawback of the considered MM layout diagrams (Figure 3 and Figure 4) is that the switching of sensor 1 will occur only at the end of the working stroke (on the reverse stroke), and the switching of the sensor 2 - at the beginning of the working stroke (on the forward stroke). Accordingly, in the opposite directions of movement of the output link for each of them, the sensors are not involved in any way. At the same time, according to the operation diagram of the limit switch shown in Figure 4, it can be turned off both in the forward and reverse motion of the MM.

An important condition for such a shutdown is that the distance (L) between the points at which the end stops are located must always be greater than the distance (S) between the stops at the beginning and end of the working stroke.

So, if you choose such a universal type of microswitch, which has at least two groups of contacts: one group is normally closed, the second is normally open $(1 \mathrm{c}+1 \mathrm{p})$; and these contacts, according to their characteristics, will provide the possibility of switching both circuits with a power load and signal ones, then there is such a switching circuit and disposition (arrangement) of control stops, which will allow implementing the method of effective monitoring and control of the positioning of the MM output link using only two microswitches, instead of three. The range of microswitches available on the Russian market allows you to choose the type of device suitable for this. As an example, consider a variant of constructing a linear position sensor MM based on two microswitches of type MP1105, version 1, UHL3 [10]. This type of microswitch meets the above requirements in terms of universality $(1 \mathrm{c}+1 \mathrm{p})$ and load capacity of contact groups, and is widely used in various electrical installations of similar purpose as a limit switch.

Applying a new disposition of control stops and two universal microswitches of type MP1 105 with contact groups $(1 c+1 p)$, the function of the limit switch can be redistributed between the two existing sensors of reference points of the working stroke, as shown in Figure 5. 


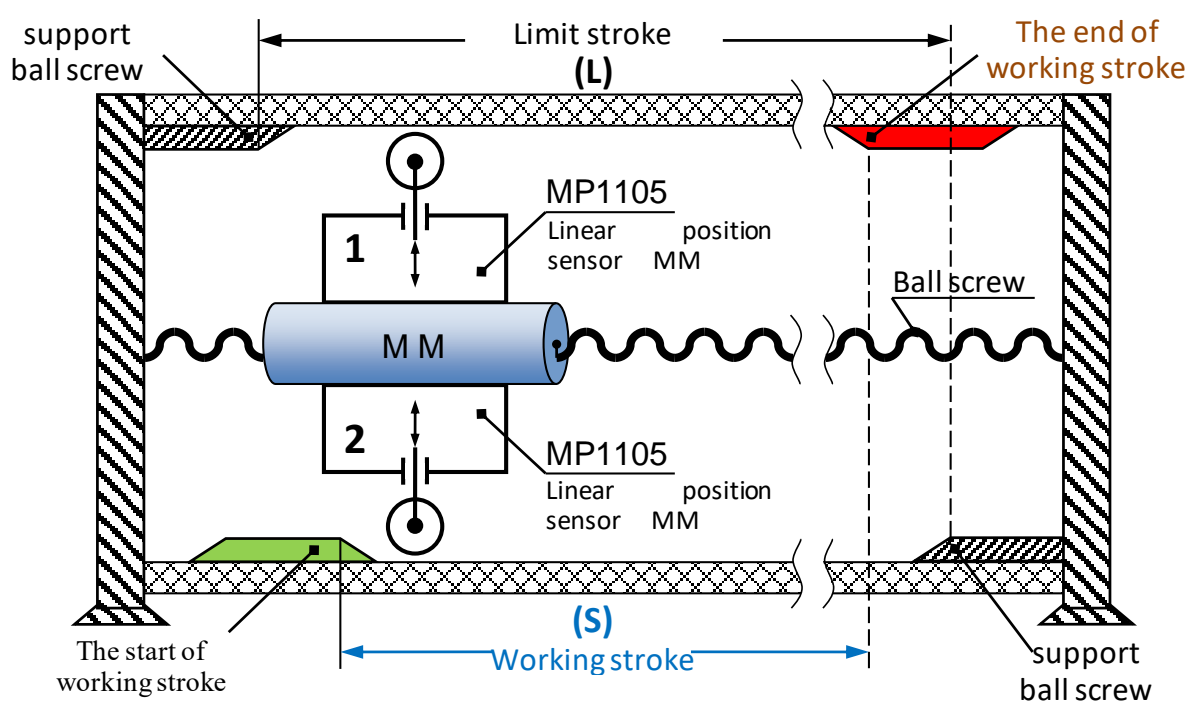

Fig. 5. A variant of the MM arrangement with two discrete sensors of the MP1105 type and a combination of control stops that perform the functions of an indicator of the starting points

Figure 5 shows that switching of each of the sensors is performed in both directions of the MM movement.

In this case, the sensor 1 on the forward stroke operates as a limit switch, and on the return stroke - as a reference point indicator "end of the working stroke". Sensor 2 on the forward stroke serves as a reference point indicator "start of the working stroke", and on the return stroke - the limit switch.

The algorithm of operation of the MM controller in accordance with the proposed method of monitoring and controlling the positioning of the MM output link is as follows. When the MM operates in the normal mode and all movements are carried out only within the limits of the working stroke, then switching of each of the sensors is possible only at the points of the beginning or end of the working stroke and only separately. Simultaneous operation of two sensors in the normal operating mode of the MM cannot occur.

If, as a result of a controller malfunction, or, for example, a malfunction of the brake mechanism of the electric motor rotating the ball screw nut, the linear movement of the MM actuator continues beyond the working stroke, then at the moment of crossing the boundary of the working stroke one of the sensors will be triggered (signaling, respectively, about "the beginning of the working stroke" or "the end of the working stroke"), and then, upon reaching the limit of the stroke, the second sensor will also necessarily work, having driven its roller pusher onto the end stop. Thus, the option of sequential operation of both sensors is possible only in an emergency situation, and according to the diagram shown in Figure 6, only in this case an emergency (emergency) break of the power supply circuit of the entire installation will occur.

The circuit for connecting two MP1105 to an electrical circuit designed by MM, which provides the required switching, both in operating modes and in emergency mode, is shown in Figure 6. 


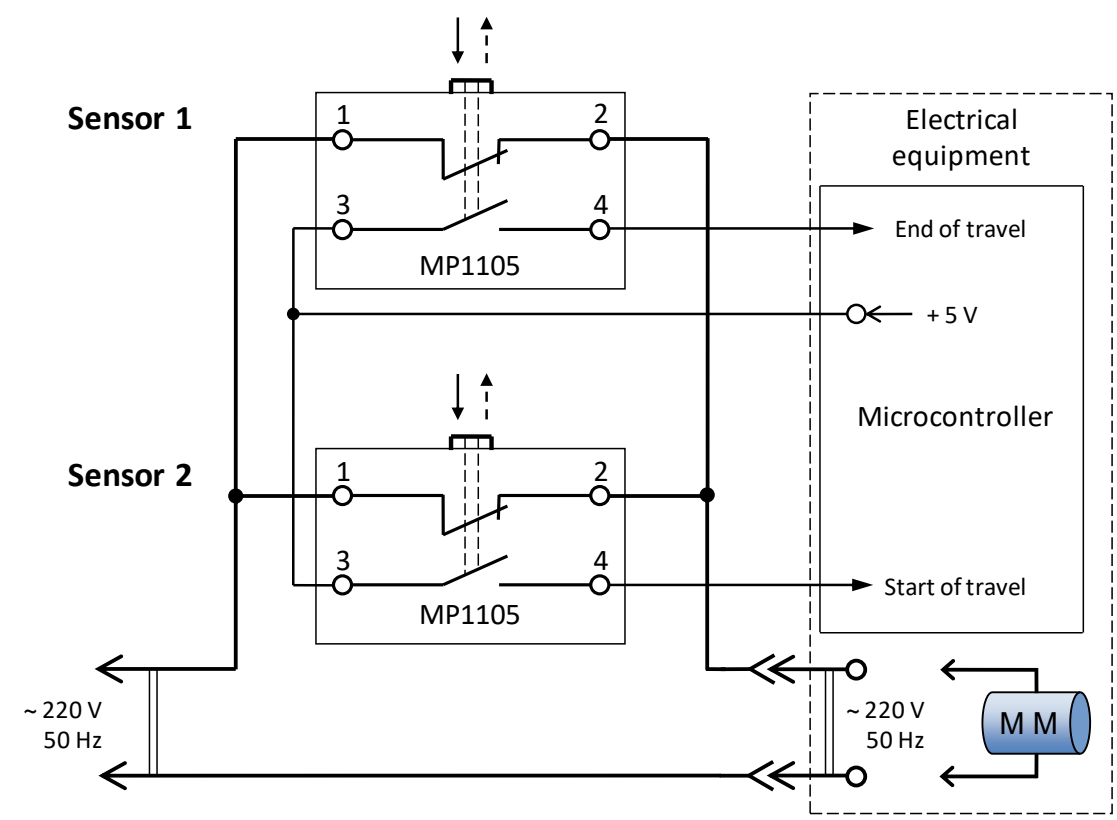

Fig. 6. Wiring diagram of microswitches type MP1105 for the MM layout with two discrete linear position sensors

Table 1. Cost comparison of sensor layout options

\begin{tabular}{|c|c|c|c|c|c|}
\hline \multicolumn{3}{|l|}{ Existing solution } & \multicolumn{3}{|l|}{ Our solution } \\
\hline Sensor Name & Quantity & $\begin{array}{l}\text { Unit price } \\
\text { in rubles }\end{array}$ & Sensor Name & Quantity & $\begin{array}{l}\text { Unit price } \\
\text { in rubles }\end{array}$ \\
\hline $\begin{array}{l}\text { Limit inductive } \\
\text { switch LJ14A3-5- } \\
\text { Z/AX }\end{array}$ & 2 & 420 & \multirow[t]{2}{*}{ MP1105 } & \multirow[t]{2}{*}{2} & \multirow[t]{2}{*}{524} \\
\hline MP1105 & 2 & 524 & & & \\
\hline \multicolumn{2}{|c|}{ Final price } & 1888 & \multicolumn{2}{|c|}{ Final price } & 1048 \\
\hline
\end{tabular}

\section{Conclusion}

Thus, an original technical solution was proposed for constructing a linear position sensor of a mechatronic module based on two discrete microswitches with an automatic "protective" power cut-off of electrical equipment when the mechatronic module goes beyond the set limits of linear motion. As a result of the economic calculations presented in table 1 , the cost of the system decreased by 46 percent. The effectiveness of monitoring and controlling the positioning of the output link of the mechatronic module lies in the use of a minimum set of sensors and switches of electrical circuits, combining the functions of the indicator of "reference points" and "limit switch" MM, which makes it possible to achieve constructive compactness and improve the weight and size characteristics of the 
MM, without losing high reliability (fault tolerance) and interference immunity of electronic equipment.

\section{References}

The effectiveness of monitoring and controlling the positioning of the output link of the mechatronic module lies in the use of a minimum set of sensors and switches of electrical circuits, combining the functions of the indicator of "reference points" and "limit switch" MM, which makes it possible to achieve constructive compactness and improve the weight and size characteristics of the MM, without losing high reliability

Efficiency control and position control mechatronic module output link is to use a minimum set of sensors and switches electric circuits combining function pointer "GCP" and "limit switch" MM thereby possible to achieve constructive compactness and improved weight and size characteristics of the MM, with no loss of high reliability (fault tolerance) and interference immunity of electronic equipment.

1. Kleanthis Thramboulidis. Challenges in the development of Mechatronic systems: The Mechatronic

Component, https://www.researchgate.net/publication/224332754_Challenges_in_the_development_ of_Mechatronic_systems_The_Mechatronic_Component

2. Artur Dyshekov, Maxim Mirzaev, Madin Shereuzhev, and Artem Peshnin. Microprocessor control system for a robotic platform for automated care for agricultural crops, https://www.e3sconferences.org/articles/e3sconf/abs/2020/77/e3sconf_ersme2020_10005/e3sconf_ersm e2020_10005.html

3. Jiajia Zhao, Mingxing Lin, Xianchun Song and Hongkui Jiang .Research on the precision loss of ball screw with short-time overload impact, https://www.researchgate.net/publication/329839082_Research_on_the_precision_loss_ of_ball_screw_with_short-time_overload_impact

4. Ball-screw transmission in the mechanisms of industrial robots / O. A. Chikhacheva, L. A. Dmitrieva, D. A. Kuznetsov, D. Yu. Formoobrazovanie / / Achievements of science: Materials of the II International Scientific Conference [Electronic resource], Karlovy Vary-Moscow, 29-30 March 2017 / Editors K. N. Voynov, I. V. Wagner, A. S. Tikhon. - Karlovy Vary-Moscow: International Center for Research Projects, 2017. - pp. 437453.

5. https://www.anaheimautomation.com/manuals/forms/encoder-guide.php (Electronic resource, last checked on 14.05.2021)

6. Gauf, A. Magnetic sensors of the angle of rotation (encoders) EcoTurn / A. Gauf, A. Guseva / / Components and technologies. - 2012. - № 1(126). - Pp. 38-41.

7. https://www.controleng.com/articles/discrete-sensors-101-sensor-types-and-bestpractices/ (Electronic resource, last checked on 14.05.2021)

8. https://realpars.com/limit-switch/ (Electronic resource, last checked on 14.05.2021)

9. https://www.ia.omron.com/support/guide/30/overview.html (Electronic resource, last checked on 14.05.2021)

10. https://asenergi.com/catalog/mikropereklyuchateli/mp-1101/mp-1105-isp-1.html (Electronic resource, last checked on 14.05.2021) 